\title{
Design of a Small Array Antenna with an Extended Cavity Structure for Wireless Power Transmission
}

\author{
Jun $\operatorname{Hur}^{1} \cdot$ Hosung $\mathrm{Choo}^{2, *}$
}

\begin{abstract}
This paper proposes a design of a small array antenna with an extended cavity structure for wireless power transmission. The proposed array element consists of a square radiating loop, which is designed to resonate at $5.8 \mathrm{GHz}$, and an extended cavity structure to reduce the mutual coupling between adjacent elements. We derive the optimal element number in a limited space by considering the input power for each element, fabrication cost, and aperture efficiency of the system. To verify the suitability of the proposed a small array with an extended cavity structure, a $2 \times 2$ downscaled array antenna is fabricated and measured in a full anechoic chamber. The results confirm that the proposed antenna element with an extended cavity structure is suitable for wireless power transmission systems; the proposed array has a transmission efficiency of about $1 \%$ at a distance of $2 \mathrm{~m}$ when the array is applied to both transmitting and receiving antennas.
\end{abstract}

Key Words: Aperture Efficiency, Extended Cavity Structure, Small Array Antenna, Wireless Power Transmission.

\section{INTRODUCTION}

Wireless power transmission (WPT) technologies have been increasingly employed in a variety of applications such as medical devices [1], mobile phones [2], and vehicle systems [3]. Microwave power transmission (MPT), which is one of the WPT technologies, has the advantage of being able to transmit power at middle and long distances [4]. This MPT method uses array antennas for both transmitting and receiving systems, and microstrip patch antennas are generally employed due to their high directivity with low-profile characteristics [5, 6]. Many previous studies, such as optimization of the array configuration, efficient feed network design, and techniques using retrodirective beamforming, have focused on the improving transmission efficiency [7-10]. However, these array antennas still exhibit the perfor- mance degradation caused by the mutual coupling between adjacent elements. Moreover, in-depth studies on the optimization of the element number in a limited small space is not sufficient yet.

In this paper, we propose the design of a small aperture array antenna for an MPT system, which is advantageous for high power transmission with a small sized array aperture. The problem of the increased mutual coupling between array elements is examined when more elements are used for a high input power of the array. We employ a 64-element array in a small space of $12 \mathrm{~cm} \times 12 \mathrm{~cm}$, and thus the input power of each element which causes the high mutual coupling and the low efficiency of the RF circuit can be minimized. The proposed array element is composed of a square loop printed on a high-dielectric substrate and an extended cavity structure enclosing the substrate. The high-

Manuscript received June 14, 2019 ; Revised August 15, 2019 ; Accepted September 11, 2019. (ID No. 20190614-047J)

${ }^{1}$ Metamaterial Electronic Device Research Center, Hongik University, Seoul, Korea.

${ }^{2}$ Department of Electronic and Electrical Engineering, Hongik University, Seoul, Korea.

"Corresponding Author: Hosung Choo (e-mail: hschoo@hongik.ac.kr)

This is an Open-Access article distributed under the terms of the Creative Commons Attribution Non-Commercial License (http://creativecommons.org/licenses/by-nc/4.0) which permits unrestricted non-commercial use, distribution, and reproduction in any medium, provided the original work is properly cited.

(c) Copyright The Korean Institute of Electromagnetic Engineering and Science. All Rights Reserved. 
dielectric substrate and loop resonator are used for miniaturization to mount multiple elements in a limited space, which enables to maximize the transmission power. The mutual coupling between adjacent elements is reduced by inserting an extended cavity structure, and the input power per each element is lowered to enhance the efficiency of the RF circuit by increasing the number of array elements. We derive the optimal number of elements in a limited space, taking into account the input power on each element, fabrication cost, and aperture efficiency of the system. To verify the performance of the optimized array for the MPT system, a $2 \times 2$ downscaled array antenna is fabricated and measured. The results demonstrate that the proposed antenna with the extended cavity structure is suitable for use as an MPT array element for high power transmission with a small sized aperture.

\section{PRoposed ARRAy ANTENNA ElEMENT}

Fig. 1 shows the geometry of the proposed MPT antenna element. The element consists of a rectangular loop and an extended cavity structure printed on a high-dielectric ceramic substrate $\left(\varepsilon_{r}=10, \tan \delta=0.004\right)$ with a thickness of $h_{1}$, which is equal to the thickness of the cavity $\left(b_{c}\right)$. The square loop is designed with the external and internal diameters of $l_{1}$ and $l_{2}$, respectively, and it is directly connected to the feed structure, which is denoted as $\left(f_{x}, f_{y}\right)$. The extended cavity structure resides above the ground plane and surrounds the entire lateral surfaces of the ceramic substrate. This proposed structure can help to decrease the mutual coupling between adjacent elements by reducing the

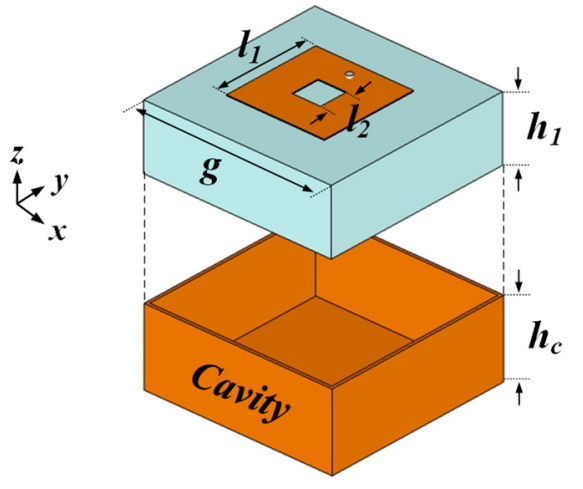

(a)

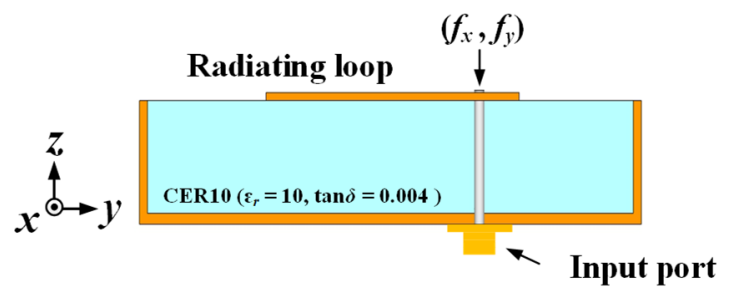

(b)

Fig. 1. Geometry of the proposed array element: (a) perspective view and (b) side view.
Table 1. Optimized values of the proposed array element

\begin{tabular}{lc}
\hline Parameter & Value \\
\hline$g$ & $15 \mathrm{~mm}$ \\
$h_{1}$ & $3.14 \mathrm{~mm}$ \\
$h_{c}$ & $3.14 \mathrm{~mm}$ \\
$l_{1}$ & $6.8 \mathrm{~mm}$ \\
$l_{2}$ & $1.5 \mathrm{~mm}$ \\
$\left(f_{x}, f_{y}\right)$ & $(0,2.1)$ \\
\hline
\end{tabular}

leakage field from the antenna substrate. The detailed design parameters are optimized by a genetic algorithm [11] and listed in Table 1.

The antenna characteristics of a fabricated element, such as the bore-sight gain and reflection coefficient, are measured in a full anechoic chamber. The measured and simulated bore-sight gains of the element are shown in Fig. 2. The dashed line indicates the simulated values, and the measured data are specified by the “ $\square$ " symbol.

Both results show a good agreement with the simulated value of $3.3 \mathrm{dBi}$ at $5.8 \mathrm{GHz}$ and $3.6 \mathrm{dBi}$ of the measured value. The low gain of the small aperture element is resolved by increasing the number of small aperture elements (64-element in this work). For a given total array space of $12 \mathrm{~cm} \times 12 \mathrm{~cm}$, as we increase the number of elements, the array gain approaches the ideal gain considering the array aperture size.

Fig. 3 presents the simulated reflection coefficient compared with the measured data. The simulated and measured values are less than $-10 \mathrm{~dB}$ from $5.73 \mathrm{GHz}$ to $5.9 \mathrm{GHz}$, and the values are $-18.5 \mathrm{~dB}$ and $-14.9 \mathrm{~dB}$ at $5.8 \mathrm{GHz}$, respectively. The slight difference between the simulation and measurement at $5.9 \mathrm{GHz}$ is due to the fabrication tolerance, such as effective dielectric permittivity, and the loss tangent of the antenna substrate. The high-Q behavior of the antenna element with a narrow bandwidth may be appropriate when a single antenna is used for a

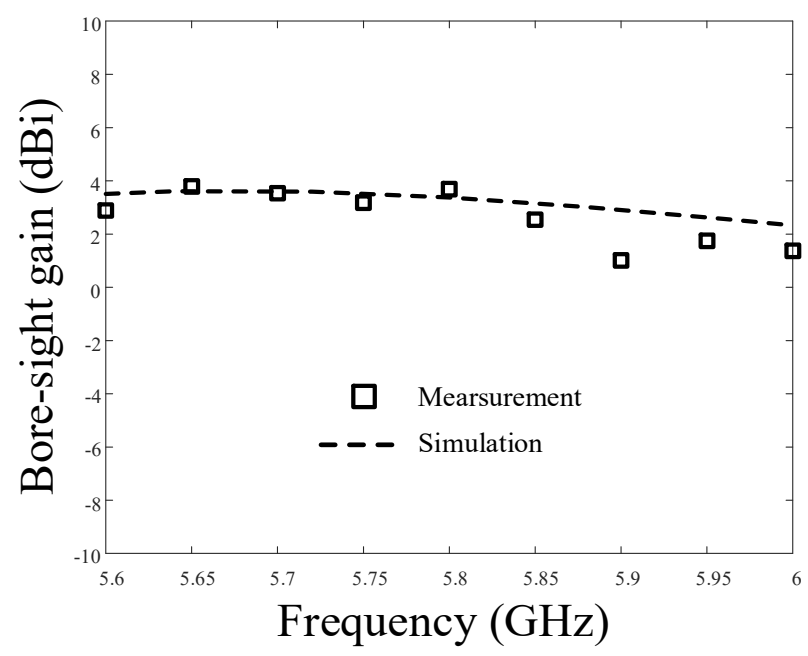

Fig. 2. Bore-sight gain of the proposed array element. 


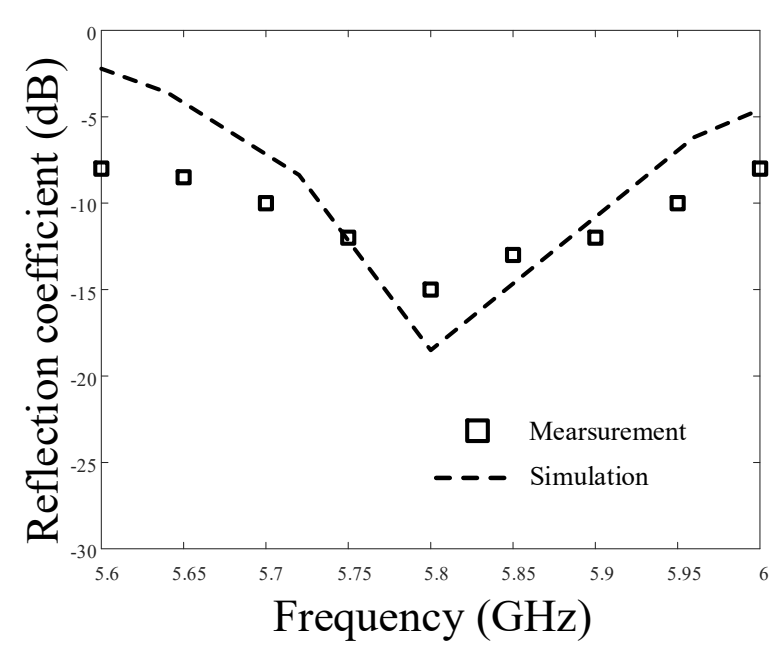

Fig. 3. Reflection coefficient of the proposed array element.

WPT system. However, the performance of the high-Q antenna can be easily degraded by an unwanted frequency shift when the elements are closely arranged for a small aperture array. This performance degradation can be minimized by using the antenna element with an appropriate Qvalue for a WPT system.

Fig. 4 shows the radiation patterns of the antenna in the $z x-$

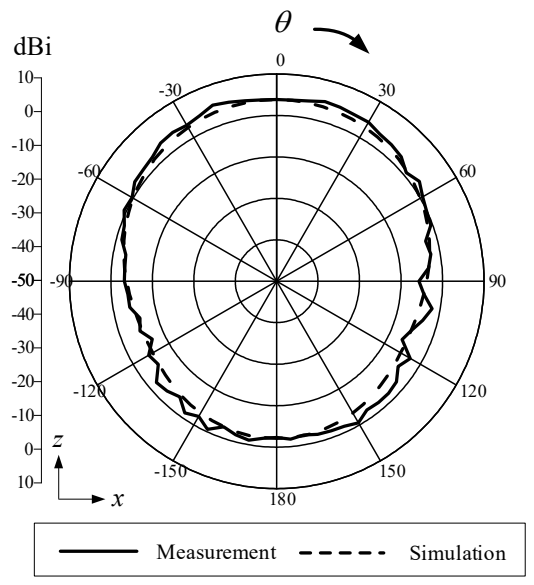

(a)

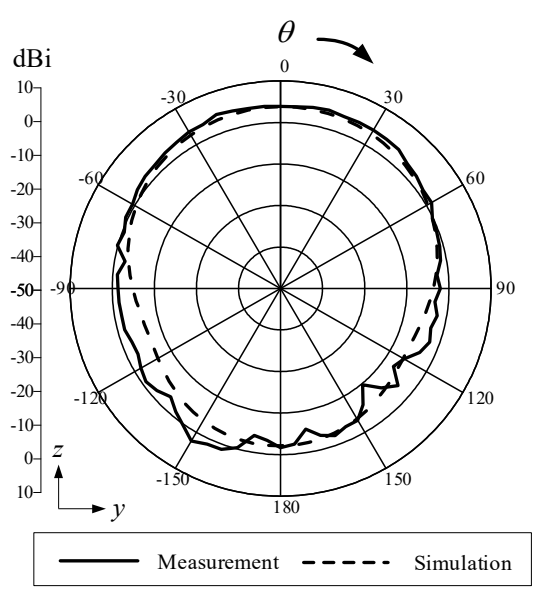

(b)

Fig. 4. Radiation patterns of the proposed array element at $5.8 \mathrm{GHz}$ : (a) $z x$-plane and (b) $z y$-plane.

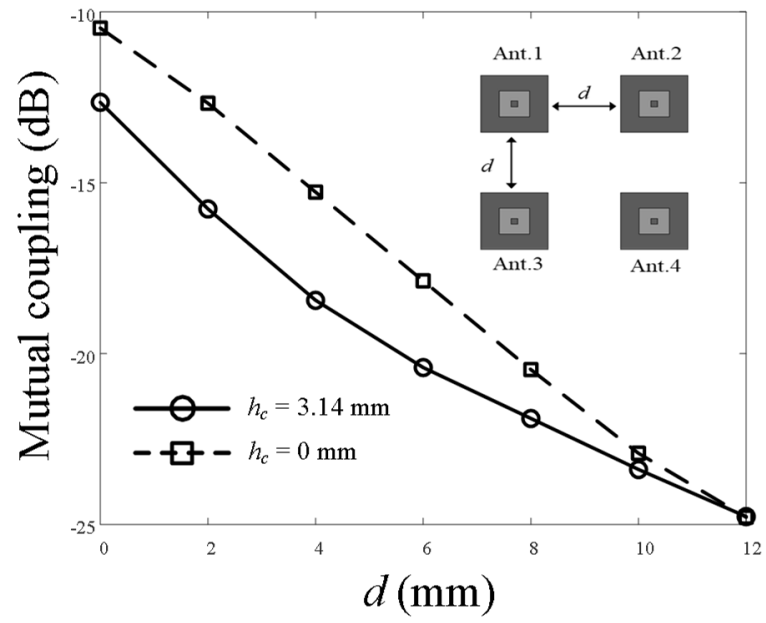

Fig. 5. Average mutual coupling according to the extended cavity height $\left(b_{c}\right)$.

plane and $z y$-plane at $5.8 \mathrm{GHz}$. The measured half-power beamwidths (HPBWs) in the $z x$ - and $z y$-planes are $120^{\circ}$ and $115^{\circ}$, respectively. As can be seen, the proposed antenna element does not have any serious pattern distortion in the upper hemisphere.

To confirm the effectiveness of the proposed extended cavity structure on the isolation characteristic, we analyze the average mutual coupling of the 4-element planar array according to the distance of the physical separation (d) from $0 \mathrm{~mm}$ to $12 \mathrm{~mm}$ as presented in Fig. 5. The antennas with and without the extended cavity structure have an identical value of $-25 \mathrm{~dB}$ at $d=12 \mathrm{~mm}$. The mutual coupling of the proposed antenna increases up to $-12.5 \mathrm{~dB}$ as $d$ becomes zero, whereas that of the antenna without the extended cavity goes up to $-10.4 \mathrm{~dB}$. The difference in the mutual coupling strength of the antenna with and without the extended cavity is greater than $2 \mathrm{~dB}$, indicating that the proposed structure can enhance the isolation characteristic.

\section{PROPOSED ARRAY ANTENNA}

The proposed element is now applied to the transmission array antenna for an MPT system. We limit the aperture size of the array to $12 \mathrm{~cm}$ to verify the feasibility of the proposed antenna with a very small aperture size. The received power is calculated using the Friis equation [12]. We assume a scenario in which the receive antenna is a $2 \times 2$ array with a gain of $7.6 \mathrm{dBi}$, and a received power of $0.2 \mathrm{~W}$ is required at a distance of $1 \mathrm{~m}$. The optimal number of elements is derived by considering the input power for each element, fabrication cost, and aperture efficiency of the array. The input power for each element is restricted to under $0.5 \mathrm{~W}$ considering the efficiency and the manufacturing cost of the RF circuits.

Fig. 6 provides a parametric study of the input power for each element $\left(\mathrm{P}_{\mathrm{i} . \mathrm{e}}\right)$ to satisfy the received power of $0.2 \mathrm{~W}$. The cost is 


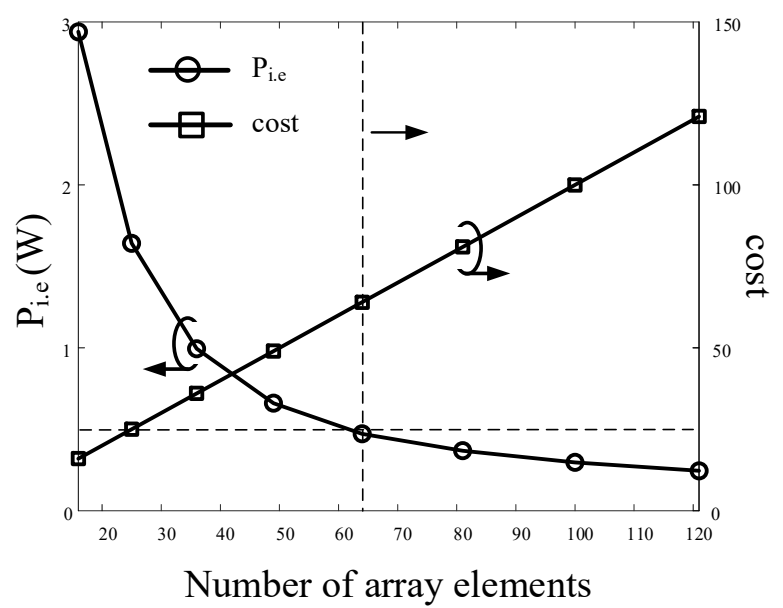

Fig. 6. Input power and cost according to the number of array elements.

the approximated total fabrication cost of the array antenna when normalizing the manufacturing cost of one antenna element to cost $=1$. The element number is required to be 64 or more to reduce the input power per element to less than $0.5 \mathrm{~W}$, which satisfies the scenario.

Fig. 7 presents the comparison of the bore-sight gain with and without the extended cavity structure according to the number of array elements. The feed structure of the power divider is not considered because we assume that there is no insertion loss of the power divider. The reason for examining the performance from a small element number to a large number of elements is to find the optimum number of array elements for maximizing the array performance in a limited space of $12 \mathrm{~cm} \times 12 \mathrm{~cm}$. The gain of the array according to the number of array elements converges to about $17.7 \mathrm{dBi}$ when the array number becomes 64 or more. The reason for the convergence is that the aperture size of the array is limited to $12 \mathrm{~cm} \times 12 \mathrm{~cm}$. The maximum gain is determined by the total aperture size and aperture efficiency, and the ideal gain achievable from the aperture size is about 18.3

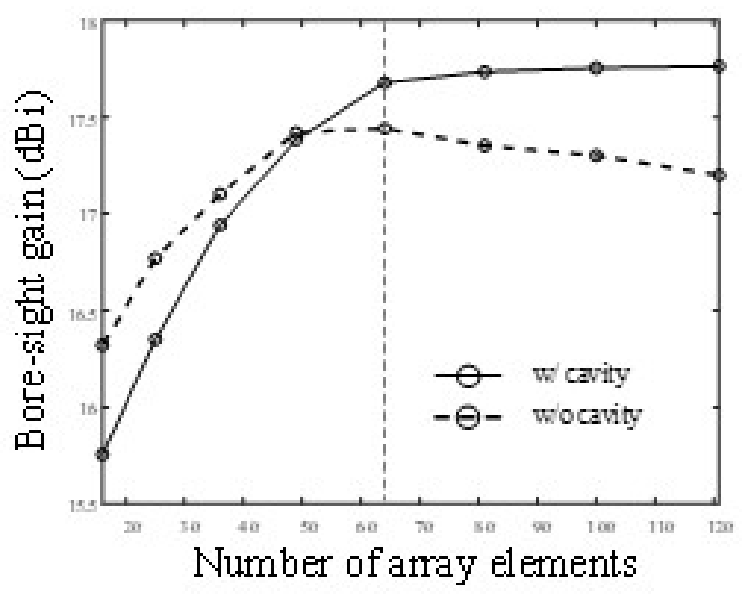

Fig. 7. Bore-sight gain according to the number of array elements.

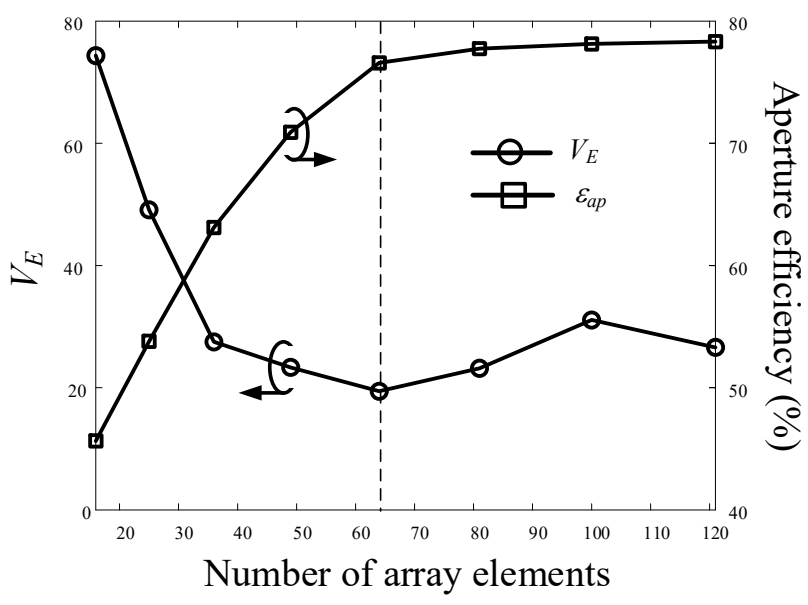

Fig. 8. Electric surface current variance and aperture efficiency according to the number of array elements.

$\mathrm{dBi}$ with an efficiency of 1 . We also compare the gain of the array without an extended cavity structure to confirm the performance enhancement by using the proposed array with an extended cavity structure. The array without an extended cavity structure has a reduced gain of $17.2 \mathrm{dBi}$ when the array number becomes 121 due to the strong mutual coupling.

To verify the performance of the proposed optimal number of elements, we analyze the aperture efficiency and electric surface current variance as shown in Fig. 8. The aperture efficiency is proportional to the antenna gain and converges to about $77 \%$ when the number of array elements is 64 . The variance for the amplitude of the electric surface current near the aperture $(-600$ $\mathrm{mm} \leq x, y \leq 600 \mathrm{~mm}, z=5 \mathrm{~mm}$ ) is examined using the Eq. (1). The variance is minimized to 20 when the aperture efficiency converges to its maximum value.

$$
V_{E}(X)=\frac{1}{n} \sum_{i=1}^{n}\left(x_{i}-u\right)^{2}, u: \text { averagevalue }
$$

Fig. 9(a) presents the geometry of the proposed 64-element array with an aperture size of $12 \mathrm{~cm} \times 12 \mathrm{~cm}$, and the simulated gains of the $z x$ - and $z y$-planes are shown in Fig. 9(b). The proposed each antenna element has a radiator with dimensions of $6.8 \mathrm{~mm} \times 6.8 \mathrm{~mm}$, and thus the antenna size is $15 \mathrm{~mm} \times 15$ $\mathrm{mm}$ including the extended cavity structure. The feed to feed distances are set to be $15 \mathrm{~mm}$, and the total array aperture size is $120 \mathrm{~mm} \times 120 \mathrm{~mm}$ with the $8 \times 8$ array configuration. Therefore, a size reduction of about 0.1 is obtained compared with a conventional $0.5 \lambda$ distance array structure $(413 \mathrm{~mm} \times 413 \mathrm{~mm})$. The simulated bore-sight gain $\left(\theta=0^{\circ}\right)$ is $17.68 \mathrm{dBi}$ at $5.8 \mathrm{GHz}$, and the HPBW in the $z x$ - and $z y$-planes are $21.28^{\circ}$ and $21.56^{\circ}$, respectively. The proposed array has a $5.6 \%$ transmission efficiency at a distance of $1 \mathrm{~m}$ when the proposed array is applied to both transmitting and receiving antennas. The transmission 


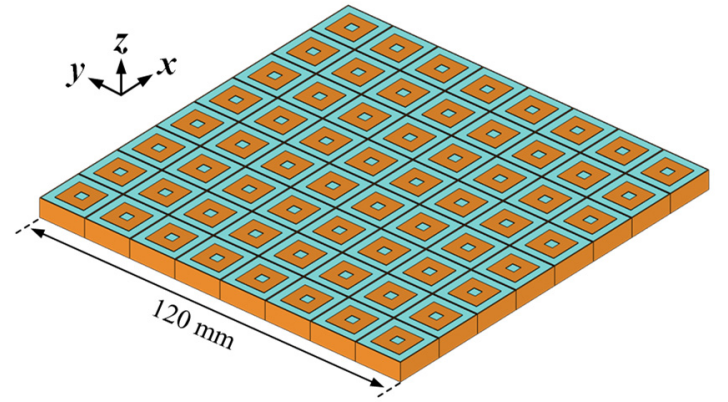

(a)

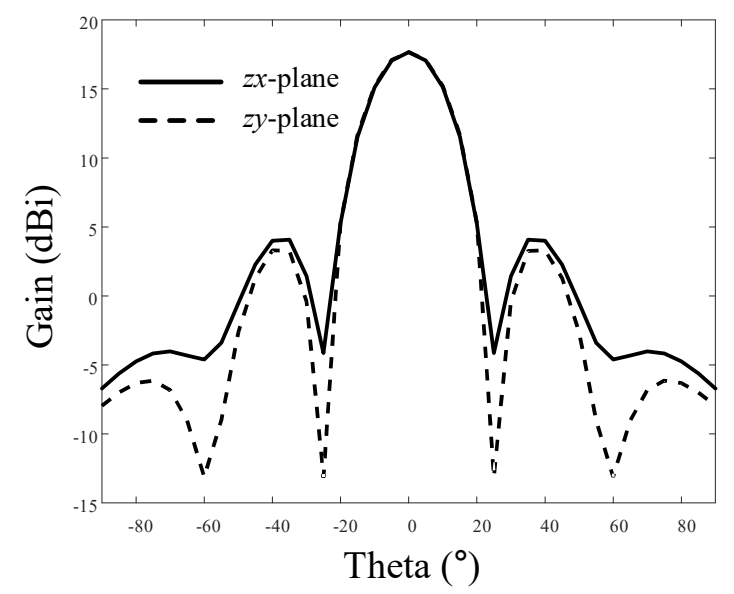

(b)

Fig. 9. Geometry and gain of the proposed $8 \times 8$ array: (a) geometry and (b) simulated gain.

efficiency of $5.6 \%$ is the antenna to antenna transmission efficiency calculated using the Friis equation. In the efficiency calculation method, we assume that there is no insertion loss in the power divider and the other RF circuits. However, the efficiency using the Friis equation may not be accurate since the analysis distance is slightly less than the far field distance. We also added the near-field simulation results of $S_{12}$ which including both the transmitting array antenna and the receiving array antenna in the simulation as listed in Table 2. The near-field simulated transmission efficiency of the proposed MPT system is $2.1 \%$ at a distance of $1 \mathrm{~m}$. The Friis equation and the fully simulated $S_{12}$ results gradually show an agreement when the distance approaches to the farfield region.

To verify the feasibility of the proposed 64-element array, a $2 \times 2$ downscaled array is fabricated and measured in a full-

Table 2. Transmission efficiency according to the distance

\begin{tabular}{ccc}
\hline \multirow{2}{*}{ Distance $(\mathrm{m})$} & \multicolumn{2}{c}{ Transmission efficiency $(\%)$} \\
\cline { 2 - 3 } & Friis equation & $S_{12}$ \\
\hline 1 & 5.6 & 2.1 \\
1.5 & 2.5 & 1.4 \\
2 & 1.4 & 1.1 \\
2.5 & 0.9 & 0.8 \\
3 & 0.6 & 0.6 \\
\hline
\end{tabular}

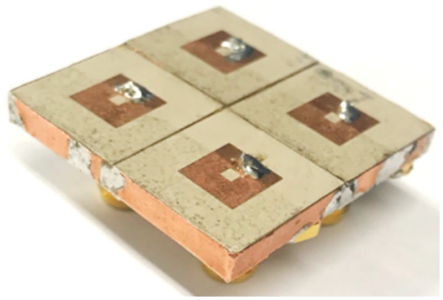

(a)

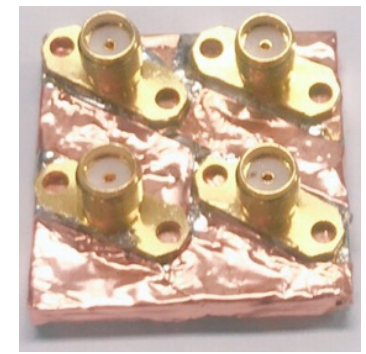

(b)

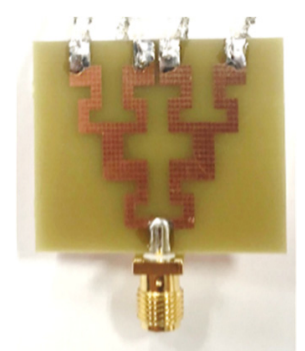

(c)

Fig. 10. Photograph of the fabricated $2 \times 2$ array: (a) perspective view, (b) bottom view, and (c) power divider.

anechoic chamber. Fig. 10(a) and (b) show the perspective and bottom views of the fabricated $2 \times 2$ downscaled array, respectively. Each array element is fed by a SMA connector, and these 4 ports are connected to a $1 \times 4$ power divider printed on a FR4 $\left(\varepsilon_{r}=4.3, \tan \delta=0.0035\right)$ substrate of $33 \mathrm{~mm} \times 29 \mathrm{~mm} \times 1.6$ $\mathrm{mm}$. The measured and simulated radiation patterns of the fabricated array in $z x$ - and $z y$-planes are indicated by the solid and dashed lines as shown in Fig. 11(a) and (b), respectively. As can be seen, the measured values agree well with the simulated results, and the array does not have any significant pattern distortion in the upper hemisphere. The transmission efficiency of the fabricated $2 \times 2$ array is $1 \%$ at a distance of $1 \mathrm{~m}$ when the $2 \times 2$ array is employed to both transmitting and receiving antennas.

\section{CONCLUSION}

The design of a small array antenna with an extended cavity structure for WPT applications was investigated. The isolation characteristic was improved to more than $2 \mathrm{~dB}$ by inserting the extended cavity structure compared with the antenna without the cavity. The optimum element number of the array considering the input power for each element, fabrication cost, and aperture efficiency was determined to be 64 . When the proposed array was applied to both transmitting and receiving antennas, a 


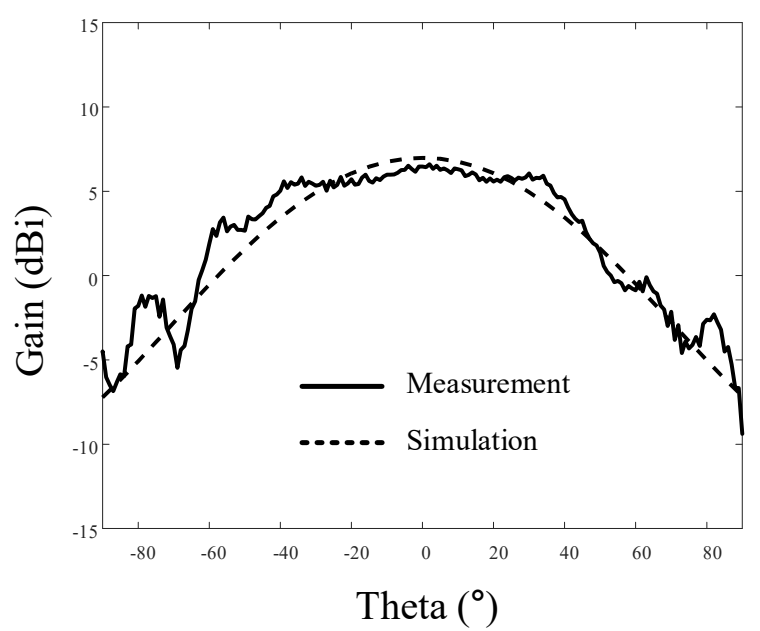

(a)

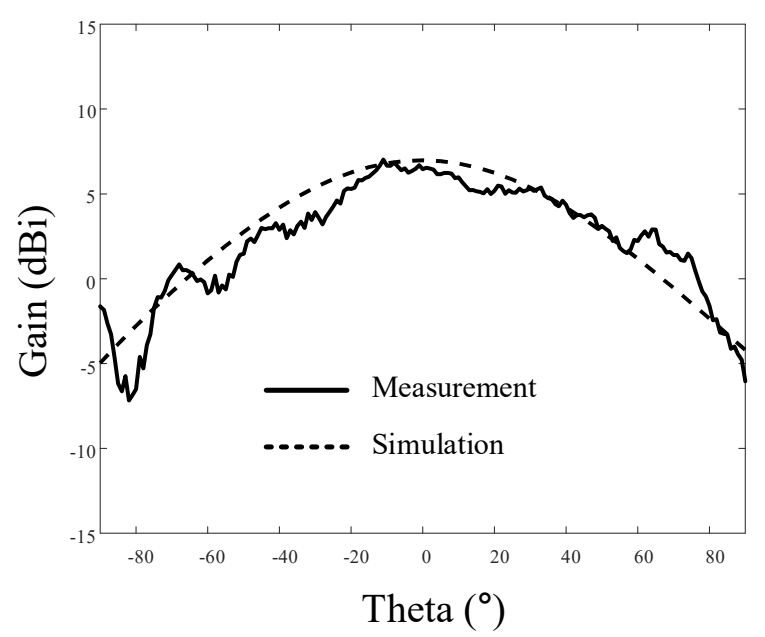

(b)

Fig. 11. Gain of the $2 \times 2$ downscaled array: (a) $z x$-plane and (b) $z y$-plane.

transmission efficiency of about $1 \%$ was observed at a distance of $2 \mathrm{~m}$. The results demonstrate that the proposed small array with an extended cavity structure is suitable for wireless power transmission applications.

This work was supported by the Basic Science Research Program through the National Research Foundation of Korea (NRF) funded by the Ministry of Education (No. 2015R1A6A1A03031833) and the NRF grant funded by the Korea government (No. NRF-2017R1A5A1015596; NRF2017R1D1A1B04031890).

\section{REFERENCES}

[1] X. Li, C. Y. Tsui, and W. H. Ki, "A 13.56 MHz wireless power transfer system with reconfigurable resonant regulating rectifier and wireless power control for implantable medical devices," IEEE Journal of Solid-State Circuits, vol. 50, no. 4, pp. 978-989, 2015.

[2] J. C. Lin, "Wireless power transfer for mobile applications, and health effects," IEEE Antennas and Propagation Magazine, vol. 55, no. 2, pp. 250-253, 2003.

[3] D. Bavastro, A. Canova, V. Cirimele, F. Freschi, L. Giaccone, P. Guglielmi, and M. Repetto, "Design of wireless power transmission for a charge while driving system," IEEE Transactions on Magnetics, vol. 50, no. 2, pp. 965-968, 2014.

[4] A. Massa, G. Oliveri, F. Viani, and P. Rocca, "Array designs for long-distance wireless power transmission: state-of-theart and innovative solutions," Proceedings of the IEEE, vol. 101, no. 6, pp. 1464-1481, 2013.

[5] J. H. Kim and B. G. Kim, "Effect of feed substrate thickness on the bandwidth and radiation characteristics of an aperture-coupled microstrip antenna with a high permittivity feed substrate," Journal of Electromagnetic Engineering and Science, vol. 18, no. 2, pp. 101-107, 2018.

[6] S. J. Yoon and J. Choi, "A low-profile broadband array antenna for home repeater applications," Journal of Electromagnetic Engineering and Science, vol. 18, no. 4, pp. 261-266, 2018.

[7] X. Yang, W. Geyi, and H. Sun, "Optimum design of wireless power transmission system using microstrip patch antenna arrays," IEEE Antennas and Wireless Propagation Letters, vol. 16, pp. 1824-1827, 2017.

[8] H. Y. Zhang, F. S. Zhang, F. Zhang, F. K. Sun, and Xie, G. J. (2017). High-power array antenna based on phase-adjustable array element for wireless power transmission. IEEE Antennas and Wireless Propagation Letters, 16, 22492253.

[9] C. T. Rodenbeck and K. Chang, "A limitation on the smallscale demonstration of retrodirective microwave power transmission from the solar power satellite," IEEE Antennas and Propagation Magazine, vol. 47, no. 4, pp. 67-72, 2005.

[10] N. Shinohara, "Beam control technologies with a high-efficiency phased array for microwave power transmission in Japan," Proceedings of the IEEE, vol. 101, no. 6, pp. 1448-1463, 2013.

[11] Y. Rahmat-Samii and E. Michielssen, Electromagnetic Optimization by Genetic Algorithms. New York, NY: John Wiley \& Sons, 1999.

[12] W. L. Stutzman and G. A. Thiele, Antenna Theory and Design. New York, NY: John Wiley \& Sons, 1981. 


\section{Jun Hur}

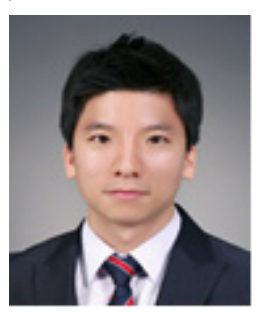

received his B.S., M.S., and Ph.D. degrees in electronic and electrical engineering from Hongik University, Seoul, Korea, in 2014, 2016, and 2019, respectively. He is currently working toward the post-doctoral researcher in the Metamaterial Electronic Device Research Center at Hongik University, Seoul, Korea. His research interests include the global positioning system antennas, antenna arrays, and position optimization of array elements for adaptive beamforming.

\section{Hosung Choo}

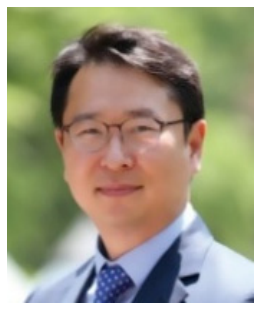

received the B.S. degree in radio science and engineering from Hanyang University in Seoul in 1998, and the M.S. and Ph.D. degrees in electrical and computer engineering from the University of Texas at Austin, in 2000 and 2003, respectively. In September 2003, he joined the school of electronic and electrical engineering, Hongik University, Seoul, Korea, where he is currently a professor. His principal areas of research are the use of the optimization algorithm in developing antennas and microwave absorbers. His studies include the design of small antennas for wireless communications, array antennas for radars, reader and tag antennas for RFID, and on-glass and conformal antennas for vehicles and aircraft. 